\title{
Can effective four-dimensional scalar theory be asymptotically free in a spacetime with extra dimensions?
}

\author{
A. V. Kisselev $\circledast^{*}$ and V. A. Petrov ${ }^{\dagger}$ \\ Division of Theoretical Physics, A.A. Logunov Institute for High Energy Physics, \\ NRC “Kurchatov Institute," 142281 Protvino, Russia
}

(Received 22 February 2021; accepted 29 March 2021; published 27 April 2021)

\begin{abstract}
We trace what happens with asymptotically free behavior of the running coupling in $\phi^{3}$ theory in six-dimensional spacetime, if to compactify two spatial dimensions on a 2D closed manifold. The result can be considered as an effective 4D theory of infinitely many KK-type scalar fields with triple interactions. The effective dimensional coupling constant inherits running to zero at high mass scales in a modified form depending on the size of the compact manifold. Some physical implications are discussed.
\end{abstract}

DOI: 10.1103/PhysRevD.103.085012

\section{INTRODUCTION}

Asymptotic freedom in QCD was discovered by Gross and Wilczek [1], and independently by Politzer [2] in 1973. The asymptotically free renormalizable field theory in four dimensions necessarily involves non-Abelian gauge fields $[3,4]$. However, it is not the case if a number of spacetime dimensions $D \neq 4$. The striking examples are the $2 \mathrm{D}$ Gross-Neneu model [5] and 2D nonlinear sigma model [6]. All these theories are renormalizable and asymptotically free. A special case is the 4D $\phi^{4}$ theory with a negative coupling constant [7]. It is a common belief that its spectrum can be shown to be unbounded from below. Nevertheless, as was shown in [8], this theory may be consistent. Especially note the 6D scalar $\phi^{3}$ theory which also exhibits the property of asymptotic freedom [9]. One may ask is there any 4D effective asymptotically free theory without gauge fields? By effective theory we mean a reduced theory obtained from a higher dimensional theory after "integrating out" extra spatial coordinates. To answer our question, one has to consider theories in a spacetime with extra dimensions (EDs).

Effective field theories with one or more compact EDs are of considerable interest during the last years. In particular, in [10] the total cross section of the scattering of two light particle was calculated in the $\phi^{4}$ scalar model with a spherical compactification. In [11] one-loop order contributions from one compact universal ED to the

\footnotetext{
*vladimir.petrov@ihep.ru

alexandre.kisselev@ihep.ru
}

Published by the American Physical Society under the terms of the Creative Commons Attribution 4.0 International license. Further distribution of this work must maintain attribution to the author(s) and the published article's title, journal citation, and DOI. Funded by SCOAP . self-energy and four-point vertex functions in a $\phi^{4}$ scalar theory are given. The one-loop low-energy effective action in the $\phi^{4}$ scalar theory and scalar QED with spacetime topology $\mathbb{R}^{3,1} \otimes S^{1}$ is calculated in [12]. The decoupling of heavy $\mathrm{KK}$ modes in an Abelian Higgs model with spacetime topologies $\mathbb{R}^{3,1} \otimes S^{1}$ and $\mathbb{R}^{3,1} \otimes S^{1} / \mathbb{Z}_{2}$ is examined in [13]. The photon self-energy, the fermion self-energy, and fermion vertex function in the one-loop approximation in the context of QED with one ED are presented in [14]. In [15], the $D+1$ dimensional $\phi^{3}$ model with an arbitrary $D$ and one compact manifold is studied. The renormalizable compactification models, when a size of compact dimensions is of the order of cutoff scale, are examined in [16]. The universal extra dimensional models defined on the six-dimensional spacetime with two spatial dimensions compactified to a two-sphere orbifold $S^{2} / Z_{2}$ were studied in [17-20]. In [21] $T^{2} / Z_{2}, S^{2} / Z_{2}$, and other orbifolds were examined.

The goal of our study is to derive an effective fourdimensional $\phi^{3}$ scalar field theory in a spacetime with two compact EDs and calculate a running coupling constant in the one-loop approximation. There are three possibilities to realize a scalar theory with a power interaction $g \phi^{n}$ which has a dimensionless coupling constant $g$; see Table I. Among them only the scalar $g \phi^{3}$ theory in six dimensions is known to be asymptotically free [9] (see also [22]). That is why we will start from this theory.

The paper is organized as follows. In Sec. II we briefly review a renormalization of the $\phi^{3}$ theory in six infinite dimensions (denoted hereafter as $\phi_{6}^{3}$, with the subscript 6 indicating the spacetime dimensionality). In the next section we examine an effective $\phi^{3}$ theory in the spacetime with four infinite and two compact dimensions (referred below as $\phi_{\text {eff }}^{3}$ ) and calculate a running coupling constant. In Sec. IV we examine a dependence of our results on a 
TABLE I. The dependence of an integer power $n$ on a number of spacetime dimensions $D$ in scalar theories with an interaction $g \phi^{n}(x)$ and dimensionless coupling constant $g$.

\begin{tabular}{llll}
\hline \hline$D$ & 3 & 4 & 6 \\
$n$ & 6 & 4 & 3 \\
\hline \hline
\end{tabular}

topology of the compact dimensions. Finally, in Sec. V a scale dependence of physical observables is analyzed. Some properties of two-dimensional inhomogeneous Epstein zeta function and truncated Epstein-like zeta function are collected in Appendix.

\section{II. $\phi^{\mathbf{3}}$ THEORY IN SIX INFINITE DIMENSIONS}

The classical Lagrangian for the $\phi_{6}^{3}$ theory in terms of bare parameters looks like

$$
\mathcal{L}=\frac{1}{2}\left[\partial_{\mu} \phi(x)\right]^{2}-\frac{1}{2} m_{0}^{2} \phi^{2}(x)-\frac{g_{0}}{3 !} \phi^{3}(x),
$$

where the bare coupling constant $g_{0}$ has a dimensionality of mass. On a classical level a cubic potential of the $\phi^{3}$ theory is not bounded below. As a consequence, there cannot be a stable ground state. However, it is not the case, if one consider the theory on a quantum level and takes into account a kinetic term in a Hamiltonian, along with the cubic and quadratic ones [23]. In terms of the renormalized $(R)$ field $\phi_{\mathrm{R}}$, mass $m$, and coupling $g$ the Lagragian is given by

$$
\mathcal{L}=\mathcal{L}_{\mathrm{R}}+\mathcal{L}_{\mathrm{CT}}
$$

where

$$
\mathcal{L}_{\mathrm{R}}=\frac{1}{2}\left[\partial_{\mu} \phi_{\mathrm{R}}(x)\right]^{2}-\frac{1}{2} m^{2} \phi_{\mathrm{R}}^{2}(x)-\frac{g}{3 !} \phi_{\mathrm{R}}^{3}(x)
$$

is its renormalized part, and the counterterm part of (2) is of the form

$$
\begin{aligned}
\mathcal{L}_{\mathrm{CT}}= & \frac{1}{2}\left(Z_{\phi}-1\right)\left[\partial_{\mu} \phi_{\mathrm{R}}(x)\right]^{2}-\frac{1}{2} \delta m^{2} \phi_{\mathrm{R}}^{2}(x) \\
& -\left(Z_{\Gamma}-1\right) \frac{g}{3 !} \phi_{\mathrm{R}}^{3}(x) .
\end{aligned}
$$

The Feynman rules are $i /\left(p^{2}-m^{2}\right)$ for a scalar propagator, and $(-i g)$ for a three-particle vertex. Let $\Gamma^{(n)}\left(p_{1}, p_{2}, \ldots p_{n-1}\right)$ be one-particle irreducible (OPI) Green's function. The inverse propagator is given by

$$
S^{-1}\left(p^{2}\right)=-i\left[p^{2}-m^{2}+\Sigma\left(p^{2}\right)\right]=-i \Gamma^{(2)}\left(p^{2}\right),
$$

where $\Sigma\left(p^{2}\right)$ is a self-energy. $\Gamma^{(3)}(p, q)$ is a three-particle vertex with "amputated" external legs.
The renormalized quantities $\left(\phi_{\mathrm{R}}, g, m\right)$ are related with the bare quantities $\left(\phi, g_{0}, m_{0}\right)$ through renormalization constants (see, for instance, [22]). In particular, the scalar field is renormalized as

$$
\phi_{\mathrm{R}}=Z_{\phi}^{-1 / 2} \phi
$$

The mass renormalization looks like

$$
m^{2}=Z_{m}^{-1} m_{0}^{2}
$$

The renormalization of the coupling constant is given by

$$
g=Z_{\phi}^{3 / 2} Z_{\Gamma}^{-1} g_{0}
$$

If we express in (2) all the parameters in terms of the bare quantities using Eqs. (6)-(8), we come to (1).

In our study, we use the dimensional regularization [24] for Feynman integrals, and the MOM scheme with the Euclidean normalization point $-\mu^{2}\left(\mu^{2}>0\right)$ for the renormalization procedure. Usually an on-shell condition is imposed on propagators and vertices of scalar fields. In massive theories where the zero momentum lies in the analyticity domain, a subtraction point $p^{2}=0$ is used [22]. Nevertheless, it is more appropriate for us to normalize OPI Green's functions at some Euclidean point, as it is done in QCD [1,25], where quarks and gluons are confined, and, consequently, have no pole masses.

The beta function of the $\phi_{6}^{3}$ theory,

$$
\beta[g(\mu)]=\mu \frac{d g(\mu)}{d \mu},
$$

is known to be $[9,22,26]$

$$
\beta(g)=-\beta_{0} g^{3}+\mathrm{O}\left(g^{5}\right),
$$

where

$$
\beta_{0}=\frac{3}{4(4 \pi)^{3}} .
$$

It is calculated up to five loops [27]. All known terms in an expansion of $\beta(g)$ are negative. Since $\beta_{0}>0$, there is the asymptotic freedom in $\phi_{6}^{3}$ theory, and

$$
\alpha(\mu)=\frac{\alpha\left(\mu_{0}\right)}{1+\frac{3}{4} \alpha\left(\mu_{0}\right) \ln \left(\mu^{2} / \mu_{0}^{2}\right)},
$$

where

$$
\alpha=\frac{g^{2}}{(4 \pi)^{3}}
$$


Note that, instead of using Eq. (9), the $\beta$ function can be alternatively defined as

$$
\beta[g(\bar{\mu})]=-\bar{\mu} \frac{d g(\bar{\mu})}{d \bar{\mu}},
$$

where $\bar{\mu}$ is a scale needed to preserve the canonical dimension of the coupling constant in the dimensional regularization. The reason is that the renormalization constants $Z_{\phi}$ and $Z_{\Gamma}$ depend on the ratio $\mu / \bar{\mu}$.

\section{III. $\phi^{3}$ THEORY IN SPACETIMES WITH TWO EXTRA COMPACT DIMENSIONS}

Let us consider $\phi^{3}$ theory in a spacetime with two extra coordinates $y_{1}, y_{2}$, and metric tensor

$$
G_{M N}=\left(1,-1,-1,-1, \eta_{m n}\right)=\left(\gamma_{\mu \nu}, \eta_{m n}\right),
$$

where $M, N=(\mu, m), \mu=0,1,2,3, m=1,2$, and $\eta_{m n}$ stands for the metric tensor of a $2 \mathrm{D}$ compact manifold. The scalar field $\phi(x, y)$ is assumed to be defined on a manifold $M_{4} \otimes T^{2} / Z_{2}$ with equal compactification radii $R_{c}$. Thus, the field fulfills the periodicity and parity conditions

$$
\begin{aligned}
& \phi(x, y)=\phi\left(x, y+2 \pi R_{c}\right), \\
& \phi(x, y)=\phi(x,-y),
\end{aligned}
$$

where $y=\left(y_{1}, y_{2}\right)$. A manifold with another topology will be considered in Sec. IV.

The action in six dimensions with two compact dimensions is given by the following expression:

$$
\begin{aligned}
\mathcal{S}_{4+[2]}= & \int d^{4} x \int_{-\pi R_{c}}^{\pi R_{c}} d y_{1} \\
& \times \int_{-\pi R_{c}}^{\pi R_{c}} d y_{2} \sqrt{-G}\left[\frac{1}{2} \partial_{M} \phi(x, y) \partial^{M} \phi(x, y)\right. \\
& \left.-\frac{1}{2} m^{2} \phi^{2}(x, y)-\frac{g}{3 !} \phi^{3}(x, y)\right],
\end{aligned}
$$

where $G=\operatorname{det}\left(G_{M N}\right)$. The canonical dimension of $\phi(x, y)$ is equal to 2. The coupling constant $g$ is dimensionless. It is clear that in the limit $R_{c} \rightarrow \infty$ the action (17) becomes a 6D action of a scalar field with interaction $g \phi^{3}$ in six infinite spacetime dimensions (see the previous section).

We can use the following Fourier expansion of the field

$$
\phi(x, y)=\frac{1}{2 \pi R_{c}} \sum_{n_{1}=-\infty}^{\infty} \sum_{n_{2}=-\infty}^{\infty} e^{i\left(n_{1} y_{1}+n_{2} y_{2}\right) / R_{c}} \phi_{n}(x),
$$

where $n=\left(n_{1}, n_{2}\right)$. Correspondingly, we have $\phi_{n}(x)=\frac{1}{2 \pi R_{c}} \int_{-\pi R_{c}}^{\pi R_{c}} d y_{1} \int_{-\pi R_{c}}^{\pi R_{c}} d y_{2} e^{-i\left(n_{1} y_{1}+n_{2} y_{2}\right) / R_{c}} \phi(x, y)$.

Note that every KK mode has canonical dimension 1.

If we require that the Kaluza-Klein $(\mathrm{KK})$ modes $\phi_{n}(x)$ are normalized,

$$
\int d^{4} x \phi_{n}(x) \phi_{n^{\prime}}(x)=\delta_{n, n^{\prime}}
$$

then

$$
\int d^{4} x \int d^{2} y \phi_{n}(x, y) \phi_{n^{\prime}}^{*}(x, y)=\delta_{n, n^{\prime}}
$$

The masses of the KK excitations are

$$
m_{n}^{2}=m_{0}^{2}+\frac{n^{2}}{R_{c}^{2}}
$$

where $n^{2}=n_{1}^{2}+n_{2}^{2}$, and $m_{0}$ means zero mode mass. Thus, the effective $4 \mathrm{D}$ action is given by

$$
\begin{aligned}
\mathcal{S}_{4 \mathrm{eff}}= & \int d^{4} x \sqrt{-\gamma}\left\{\frac{1}{2} \partial_{\mu} \phi_{0}(x) \partial^{\mu} \phi_{0}(x)-\frac{1}{2} m_{0}^{2} \phi_{0}^{2}(x)\right. \\
& -\sum_{n \neq 0}\left[\frac{1}{2} \partial_{\mu} \phi_{n}(x) \partial^{\mu} \phi_{n}(x)-\frac{1}{2} m_{n}^{2} \phi_{n}^{2}(x)\right] \\
& -\frac{g_{4}}{3 !}\left[\phi_{0}^{3}(x)+\phi_{0}(x) \sum_{n \neq 0} \phi_{n}(x) \phi_{-n}(x)\right. \\
& \left.\left.+\sum_{n, m, k \neq 0} \phi_{n}(x) \phi_{m}(x) \phi_{k}(x) \delta_{n+m+k, 0}\right]\right\}
\end{aligned}
$$

where $\gamma=\operatorname{det}\left(\gamma_{\mu \nu}\right)$. Here

$$
g_{4}=\frac{g}{2 \pi R_{c}}
$$

is an effective four-dimensional coupling constant. Thus, it is the inverse compactification scale $R_{c}^{-1}$ that makes $g_{4}$ a quantity with the dimension of mass.

\section{A. Effective four-dimensional propagator in one-loop approximation}

One of our main goals is a calculation of a scale dependence of the coupling constant $g_{4}$ (24). As one can see from (23), it is the same for zero mode interactions, interactions between zero and $\mathrm{KK}$ modes, and nonzero mode interactions. That is why, here and in what follows it is assumed that all external particles have zero $\mathrm{KK}$ numbers. From the very beginning, we put $m_{0}=0$. 
The four-dimensional self-energy of the scalar field at order $\mathrm{O}\left(g^{2}\right)$ is given by the diagram in Fig. 1. It can be divided into two parts

$$
\Sigma\left(p^{2}\right)=\Sigma_{0}\left(p^{2}\right)+\Sigma_{\mathrm{KK}}\left(p^{2}\right)
$$

where

$\Sigma_{0}\left(p^{2}\right)=-\frac{i}{2} g_{4}^{2} \bar{\mu}^{2 \epsilon} \int_{0}^{1} d x \int \frac{d^{D} k}{(2 \pi)^{D}} \frac{1}{\left[k^{2}+p^{2} x(1-x)\right]^{2}}$

is the contribution from zero mode, and

$$
\begin{aligned}
& \Sigma_{\mathrm{KK}}\left(p^{2}\right) \\
& \quad=-\frac{i}{2} g_{4}^{2} \bar{\mu}^{2 \epsilon} \sum_{n \neq 0} \int_{0}^{1} d x \int \frac{d^{D} k}{(2 \pi)^{D}} \frac{1}{\left[k^{2}+p^{2} x(1-x)-m_{n}^{2}\right]^{2}}
\end{aligned}
$$

is the contribution from $\mathrm{KK}$ massive modes. It is assumed that $p^{2}<0$. We define

$$
D=4-2 \varepsilon
$$

We find that

$$
\begin{aligned}
\Sigma_{0}\left(p^{2}\right) & =\frac{g_{4}^{2}}{2(4 \pi)^{2-\epsilon}} \Gamma(\epsilon)\left(\frac{\bar{\mu}^{2}}{-p^{2}}\right)^{\varepsilon} \int_{0}^{1} d x[x(1-x)]^{-\varepsilon} \\
& =\frac{\alpha}{2 \pi} R_{c}^{-2}\left[N_{\varepsilon}-\ln \frac{-p^{2}}{\bar{\mu}^{2}}+2\right]+\mathrm{O}(\varepsilon),
\end{aligned}
$$

where

$$
N_{\varepsilon}=\frac{1}{\varepsilon}-\gamma_{E}+\ln 4 \pi
$$

Thus, the zero mode contributes to the mass renormalization only.

Now we consider the contribution from the massive modes

$$
\begin{aligned}
\Sigma_{\mathrm{KK}}\left(p^{2}\right)= & -\frac{i}{2} g_{4}^{2}\left(\bar{\mu} R_{c}\right)^{2 \epsilon} R_{c}^{-2} \sum_{n_{1}, n_{2} \neq 0} \int_{0}^{1} d x \int \frac{d^{D} l}{(2 \pi)^{D}} \\
& \times \frac{1}{\left[l^{2}+p^{2} R_{c}^{2} x(1-x)-n_{1}^{2}-n_{2}^{2}\right]^{2}},
\end{aligned}
$$

where $l=k R_{c}$. Since

$$
\begin{aligned}
& \int \frac{d^{D} l}{(2 \pi)^{D}} \frac{1}{\left[l^{2}+p^{2} R_{c}^{2} x(1-x)-n_{1}^{2}-n_{2}^{2}\right]^{2}} \\
& \quad=\frac{i}{(4 \pi)^{2-\varepsilon}} \Gamma(\varepsilon)\left[-p^{2} x(1-x)+n_{1}^{2}+n_{2}^{2}\right]^{-\varepsilon},
\end{aligned}
$$

we obtain

$$
\begin{aligned}
\Sigma_{\mathrm{KK}}\left(p^{2}\right)= & \frac{\alpha}{2 \pi} \Gamma(\varepsilon)(4 \pi)^{\varepsilon}\left(\bar{\mu} R_{c}\right)^{2 \epsilon} R_{c}^{-2} \\
& \times \sum_{n_{1}, n_{2} \neq 0} \int_{0}^{1} d x\left[-p^{2} R_{c}^{2} x(1-x)+n_{1}^{2}+n_{2}^{2}\right]^{-\varepsilon} .
\end{aligned}
$$

The series in (33) converges absolutely for $\operatorname{Re} \varepsilon>1$. To define this series for other values of $\varepsilon$, we require its analytic continuation using the two-dimensional inhomogeneous Epstein zeta function $Z_{2}^{a}(s)$ [28]

$$
Z_{2}^{a}(s)=\sum_{n_{1}, n_{2} \in Z^{2}}^{\prime} \frac{1}{\left(n_{1}^{2}+n_{2}^{2}+a\right)^{s}},
$$

with $a>0$ [the prime in (34) means that the point $n=0$ is to be excluded from the sum]. The zeta function regularization method for the quantum physical systems was proposed for the first time in $[29,30]$. The Riemann zeta function $\zeta(s)$ was used in fixing a critical spacetime dimension of the string theory (see, for instance, [31]). Recently, one-dimensional inhomogeneous Epstein zeta function $Z_{1}^{a}(s)$ was applied to quantify the UV divergences induced by the KK fields [11-13]. In [14] both $Z_{1}^{a}(s)$ and $n$-dimensional inhomogeneous function $Z_{n}^{a}(s)$ were used.

In Appendix formula (A1) is presented, which gives an analytical continuation for the function $Z_{2}^{a}(s)$. It is defined on the complex plane of $s$. It has an infinite number of simple poles, but converges both in the $s \rightarrow 0$ limit, and with $a=0$. These results are a consequence of the analytical properties of the inhomogeneous Epstein zeta function.

Let us define

$$
c=-p^{2} R_{c}^{2} x(1-x) .
$$

Note that $c>0$, except for two points $x=0,1$. We obtain from (33)-(35)

$$
\Sigma_{\mathrm{KK}}\left(p^{2}\right)=\frac{\alpha}{2 \pi} \Gamma(\varepsilon)(4 \pi)^{\varepsilon}\left(\bar{\mu} R_{c}\right)^{2 \epsilon} R_{c}^{-2} \int_{0}^{1} d x Z_{2}^{c}(\varepsilon),
$$

where

$$
Z_{2}^{c}(\varepsilon)=-c^{-\varepsilon}-\frac{\pi c^{1-\varepsilon}}{1-\varepsilon}+\frac{A(\varepsilon ; c)}{\Gamma(\varepsilon)}
$$


Since $Z_{2}^{c}(a)$ is finite for $c=0$, see (A3), we can take $c>0$ for all $x \in[0,1]$. An explicit expression for $A(s ; a)$ in (37) is given by Eq. (A2). The function $A(\varepsilon ; c)$ converges, as $\varepsilon \rightarrow 0$, and, consequently, $Z_{2}^{c}(0)=-(1+\pi c)$. The KK divergence [the first term in (37)] exactly cancels the zero mode divergence (29). A similar effect was seen in the context of quantum electrodynamics with one ED [12]. Since $A(\varepsilon ; c)$ decreases exponentially as $c \rightarrow \infty$, we find for large $R_{c}$

$$
\begin{aligned}
\Sigma\left(p^{2}\right) & =p^{2} \frac{\alpha}{2} \Gamma(\varepsilon)(4 \pi)^{\varepsilon}\left(\frac{\bar{\mu}^{2}}{-p^{2}}\right)^{\varepsilon} \int_{0}^{1} d x[x(1-x)]^{1-\varepsilon} \\
& =p^{2} \frac{\alpha}{12}\left(N_{\varepsilon}-\ln \frac{-p^{2}}{\bar{\mu}^{2}}+\frac{5}{3}\right)+\mathrm{O}(\varepsilon) .
\end{aligned}
$$

As a result, for $\mu R_{c} \gg 1$, the field renormalization constant is equal to

$$
Z_{\phi}=1-\frac{\alpha}{12}\left(N_{\varepsilon}-\ln \frac{\mu^{2}}{\bar{\mu}^{2}}+\frac{5}{3}\right)
$$

It differs from the field renormalization in the $\phi_{6}^{3}$ theory by a constant term only. Note, there is no dependence on $R_{c}$ in (39). Since $\Sigma\left(p^{2}\right) \sim p^{2}$, the renormalized theory remains massless in the one-loop approximation (no mass renormalization holds).

\section{B. Effective four-dimensional vertex in one-loop approximation}

The effective four-dimensional three-point vertex $\Gamma^{(3)}$ is defined by the diagram presented in Fig. 2. It is a sum of two terms,

$$
\Gamma^{(3)}(p, q)=\Gamma_{0}^{(3)}(p, q)+\Gamma_{\mathrm{KK}}^{(3)}(p, q),
$$

where

$$
\Gamma_{0}^{(3)}(p, q)=2 g_{4}^{3} \bar{\mu}^{2 \epsilon} \int_{0}^{1} d x x \int_{0}^{1} d y \int \frac{d^{D} k}{(2 \pi)^{D}} \frac{1}{\left(k^{2}-M^{2}\right)^{3}},
$$

and

$$
\begin{aligned}
\Gamma_{\mathrm{KK}}^{(3)}(p, q) & =2 g_{4}^{3} \bar{\mu}^{2 \epsilon} \sum_{n_{1}, n_{2} \neq 0} \int_{0}^{1} d x x \int_{0}^{1} d y \int \frac{d^{D} k}{(2 \pi)^{D}} \frac{1}{\left(k^{2}-M^{2}-m_{n}^{2}\right)^{3}} \\
& =2 g_{4}^{3} \bar{\mu}^{2 \epsilon} R_{c}^{2+2 \varepsilon} \sum_{n_{1}, n_{2} \neq 0} \int_{0}^{1} d x x \int_{0}^{1} d y \int \frac{d^{D} l}{(2 \pi)^{D}} \frac{1}{\left(l^{2}-M^{2} R_{c}^{2}-n_{1}^{2}-n_{2}^{2}\right)^{3}} .
\end{aligned}
$$

Here a notation

$$
\begin{aligned}
M^{2}= & -x\left[p^{2} x y(1-y)+q^{2} y(1-x)\right. \\
& \left.+(p+q)^{2}(1-x)(1-y)\right]
\end{aligned}
$$

is introduced. We assume that $p^{2}, q^{2},(p+q)^{2}<0$. It means that $M^{2}>0$, except for two points $(x, y)=$ $(1,0),(1,1)$. The integral in (41) converges, as $\varepsilon \rightarrow 0$, and we obtain

$$
\begin{aligned}
\Gamma_{0}^{(3)}(p, q)= & \left(-i g_{4}\right) \frac{\alpha}{\pi} \Gamma(1+\varepsilon)(4 \pi)^{\varepsilon} \bar{\mu}^{2 \epsilon} R_{c}^{-2} \\
& \times \int_{0}^{1} d x x \int_{0}^{1} d y\left(M^{2}\right)^{-1-\varepsilon} .
\end{aligned}
$$

In particular, we find for $\varepsilon=0$

$$
\left.\Gamma_{0}^{(3)}(p, q)\right|_{p^{2}=q^{2}=(p+q)^{2}=-\mu^{2}}=\left(-i g_{4}\right) \frac{\alpha}{2 \pi} B\left(\mu R_{c}\right)^{-2},
$$

where

$$
\begin{aligned}
B & =2 \int_{0}^{1} d x \int_{0}^{1} d y[1-x+x y(1-y)]^{-1} \\
& =\frac{1}{27}\left[\psi_{1}\left(\frac{1}{3}\right)+\psi_{1}\left(\frac{1}{6}\right)-\psi_{1}\left(\frac{5}{6}\right)-\psi_{1}\left(\frac{2}{3}\right)\right],
\end{aligned}
$$

$\psi_{1}(z)=\left(d^{2} / d z^{2}\right) \ln \Gamma(z)$ being the trigamma function [32].

The integral on the right-hand side of Eq. (42) is equal to

$$
\begin{aligned}
& \int \frac{d^{D} l}{(2 \pi)^{D}} \frac{1}{\left(l^{2}-M^{2} R_{c}^{2}-n_{1}^{2}-n_{2}^{2}\right)^{3}} \\
& \quad=-\frac{i}{2(4 \pi)^{2-\varepsilon}} \Gamma(1+\varepsilon)\left(M^{2} R_{c}^{2}+n_{1}^{2}+n_{2}^{2}\right)^{-1-\varepsilon}
\end{aligned}
$$

that results in

$$
\begin{aligned}
\Gamma_{\mathrm{KK}}^{(3)}(p, q)= & \left(-i g_{4}\right) \frac{\alpha}{\pi} \Gamma(1+\varepsilon)(4 \pi)^{\varepsilon}\left(\bar{\mu} R_{c}\right)^{2 \epsilon} \\
& \times \int_{0}^{1} d x x \int_{0}^{1} d y Z_{2}^{M^{2} R_{c}^{2}}(1+\varepsilon) .
\end{aligned}
$$

Thus, the infinite number of UV divergences results in the two-dimensional inhomogeneous Epstein zeta function. We find from Eqs. (A1), (A2) 


$$
\begin{aligned}
Z_{2}^{M^{2} R_{c}^{2}}(1+\varepsilon)= & -\left(M^{2} R_{c}^{2}\right)^{-1-\varepsilon}+\frac{\pi\left(M^{2} R_{c}^{2}\right)^{-\varepsilon}}{\varepsilon} \\
& +\frac{A\left(1+\varepsilon ; M^{2} R_{c}^{2}\right)}{\Gamma(1+\varepsilon)}
\end{aligned}
$$

with $A(1 ; c)$ being a finite quantity. As one can see from (49), $Z_{2}^{M^{2} R_{c}^{2}}(1+\varepsilon)$ has a simple pole at $\varepsilon=0$. It can be easily shown that in the limit $\varepsilon \rightarrow 0$ the fist term in (49), after substitution in (48), cancels the zero mode contribution (44), and we obtain

$$
\begin{aligned}
\Gamma^{(3)}(p, q)= & \left(-i g_{4}\right) \frac{\alpha}{\pi} \Gamma(1+\varepsilon)(4 \pi)^{\varepsilon}\left(\bar{\mu} R_{c}\right)^{2 \epsilon} \\
& \times \int_{0}^{1} d x x \int_{0}^{1} d y\left[\frac{\pi\left(M^{2} R_{c}^{2}\right)^{-\varepsilon}}{\varepsilon}+\frac{A\left(1+\varepsilon ; M^{2} R_{c}^{2}\right)}{\Gamma(1+\varepsilon)}\right] .
\end{aligned}
$$

Thus, for $\varepsilon \rightarrow 0$ the vertex renormalization constant is given by the expression

$$
\begin{aligned}
Z_{\Gamma}= & 1-\frac{\alpha}{\pi} \int_{0}^{1} d x x \int_{0}^{1} d y \\
& \times\left[\Gamma(\varepsilon) \pi(4 \pi)^{\varepsilon}\left(\frac{\bar{\mu}^{2}}{M_{\mu}^{2}}\right)^{\varepsilon}+A\left(1 ; M_{\mu}^{2} R_{c}^{2}\right)\right],
\end{aligned}
$$

where

$$
M_{\mu}^{2}=\mu^{2} x[1-x+x y(1-y)],
$$

$-\mu^{2}$ being the renormalization point. As one can see from (51), the vertex renormalization constant $Z_{\Gamma}^{-1}$ depends both on the ratio $\mu / \bar{\mu}$ and on the compactification radius via dimensionless parameter $\mu R_{c}$. The vertex has a divergence related with a summation over KK number, while Feynman integral is finite.

However, for $\mu R_{c} \gg 1$ (and, consequently, for $M_{\mu}^{2} R_{c}^{2} \gg 1$ ), the function $A\left(1 ; M_{\mu}^{2} R_{c}^{2}\right)$ decreases exponentially [see Eq. (A2)], and we obtain

$$
Z_{\Gamma}^{-1}=1+\frac{\alpha}{2}\left[N_{\varepsilon}-\ln \left(\frac{\mu^{2}}{\bar{\mu}^{2}}\right)-C\right]
$$

where

$$
\begin{aligned}
C & =2 \int_{0}^{1} x d x \int_{0}^{1} d y\{\ln x+\ln [1-x+x y(1-y)]\} \\
& =\frac{2 B}{3}-3 .
\end{aligned}
$$

As we can see, if the compactification radius exceeds the physical scale, $R_{c} \gg \mu^{-1}$, it disappears from the renormalization constants (39) and (51).
The renormalized effective four-dimensional vertex is proportional to $g_{4}(24)$. The fact that the coupling of the four-dimensional fields becomes smaller at larger $R_{c}$ can be easily understood. As it follows from (18), the wave function of the field $\phi_{n}(x)$ in the $y$-space is given by

$$
\psi_{n}(y)=\frac{1}{2 \pi R_{c}} e^{i n y / R_{c}}
$$

The coupling constant of three fields $\phi_{n}(x), \phi_{m}(x), \phi_{k}(x)$ is defined by overlapping of their wave functions

$g \int_{-\pi R_{c}}^{\pi R_{c}} d y_{1} \int_{-\pi R_{c}}^{\pi R_{c}} d y_{2} \psi_{n}(y) \psi_{m}(y) \psi_{k}(y)=\frac{g}{2 \pi R_{c}} \delta_{n+m+k, 0}$.

It tends to zero as $R_{c}$ grows. Thus, in the limit $R_{c} \rightarrow \infty$ (all six dimensions are infinite), the $\phi_{\text {eff }}^{3}$ theory becomes a theory of a free scalar field, whose propagator is equal to that of the $\phi_{4}^{3}$ theory.

\section{Running coupling constant}

Let us consider large values of the mass scale $\mu$, namely, $\mu \gg R_{c}^{-1}$. It follows from Eqs. (8), (39), and (53) that in the one-loop approximation the beta function is equal to

$$
\beta(g)=-\frac{3 R_{c}^{2}}{64 \pi} g^{3}
$$

and, correspondingly,

$$
\mu^{2} \frac{\partial \alpha_{4}(\mu)}{\partial \mu^{2}}=-\frac{3 R_{c}^{2}}{16} \alpha_{4}^{2}(\mu)
$$

where

$$
\alpha_{4}=\frac{g_{4}^{2}}{4 \pi}
$$

Let us note, it is the dimensional variable $R_{c}^{2} \ln \left(\mu^{2} / \mu_{0}^{2}\right)$, not the dimensionless quantity $\ln \left(\mu^{2} / \mu_{0}^{2}\right)$, which should be regarded as an evolution parameter for the coupling constant $\alpha_{4}(\mu)$. It is to be expected, since the coupling $\alpha_{4}$ has dimension -2 . As a result, we obtain

$$
\begin{aligned}
\alpha_{4}(\mu) & =\frac{\alpha_{4}\left(\mu_{0}\right)}{1+\frac{3}{16} \alpha_{4}\left(\mu_{0}\right) R_{c}^{2} \ln \left(\mu^{2} / \mu_{0}^{2}\right)} \\
& =\frac{16}{3 R_{c}^{2} \ln \left(\mu^{2} / \Lambda^{2}\right)},
\end{aligned}
$$

where

$$
\begin{aligned}
\Lambda^{2} & =\mu_{0}^{2} \exp \left[-16 /\left(3 \alpha_{4}\left(\mu_{0}\right) R_{c}^{2}\right)\right] \\
& =\mu_{0}^{2} \exp \left[-4 /\left(3 \alpha\left(\mu_{0}\right)\right]\right.
\end{aligned}
$$


We remind that Eqs. (60) and (61) hold in the one-loop approximation and at $\mu \gg \Lambda$. Ghost pole at $\mu=\Lambda$ is safely eliminated if to respect the causality [33].

Thus, the effective four-dimensional scalar $\phi^{3}$ theory in the flat spacetime with two compact EDs exhibits the property of asymptotic freedom. Namely, its effective coupling constant $\alpha_{4}(\mu)$ tends logarithmically to zero, as the mass scale $\mu$ grows. One can say that four-dimensional theory does not forget its higher dimensional origin.

All this can be understood as follows. The renormalization of the coupling constant is defined by the UV divergences and renormalization scale $\mu$, and "it is not aware" of the scale $R_{c}^{-1}$, provided $\mu \gg R_{c}^{-1}$. In other words, the large scale $R_{c}$ is irrelevant to a small-distance physics. As a result, the effective four-dimensional coupling constant $g_{4}$ exhibits a large-scale behavior of the coupling constant in the $\phi_{6}^{3}$ theory. For a detailed discussion of this phenomenon, see Sec. V.

It is interesting to compare our prediction (57) with the results obtained for an effective 4D $\lambda \phi^{4}$ theory in a spacetime with one compact ED [12]. Is has been found that in such a theory an effective coupling constant in oneloop approximation is renormalized by the constant

$$
Z_{\phi}^{3 / 2} Z_{\Gamma}^{-1}=1+\frac{3 \lambda^{2}}{16 \pi^{2}}\left[\frac{1}{\varepsilon}+\ln \left(\mu R_{c}\right)\right] .
$$

Note that $\lambda=\bar{\lambda} /\left(2 \pi R_{c}\right)$, where $\bar{\lambda}$ is the coupling constant in a $5 \mathrm{D}$ action with dimension -1 . Thus, one cannot obtain a RG-like equation for $\lambda$ with respect to the scale $\bar{M}=R_{c}^{-1}$, as it is erroneously stated in [12] (see also [15]), except when $\bar{\lambda}=\bar{\lambda}\left(R_{c}\right)=$ constant $\times R_{c}$. For instance, if we assume that this relation takes place for small $R_{c}$, then we come to the equation with respect to the intrinsic scale of the spacetime topology,

$$
\frac{d \lambda}{d \ln \bar{M}}=-\frac{3 \lambda^{2}}{16 \pi^{2}},
$$

valid for large $\bar{M}$.

As for the case $\mu \ll R_{c}^{-1}$, it can be shown that $\alpha_{4}(\mu)$ tends to a constant value, as $\mu$ grows (while being less than $R_{c}^{-1}$ ). As one see from (36), the total divergence in $\varepsilon=$ $(4-D) / 2$ is due to the UV divergent Feynman integral, while the sum in the KK modes gives a finite result $\left[Z_{2}^{c}(\varepsilon)\right.$ is finite, as $\varepsilon \rightarrow 0$ ]. On the contrary, as Eq. (48) shows, the vertex divergence comes from the infinite sum over KK modes only $\left[Z_{2}^{c}(1+\varepsilon) \sim \varepsilon^{-1}\right.$, as $\left.\varepsilon \rightarrow 0\right]$, while the Feynman integral is finite. If $\mu \gg R_{c}^{-1}$, infinite and $\mu$-dependent parts of the counterterms of the origin, sixdimensional, theory and those of the reduced theory coincide. However, our calculations have shown that $\mu$-dependent parts of the renormalization constants differ for $\mu \ll R_{c}^{-1}$, and a nontrivial dependence on $R_{c}$ occurs. Let us note that the divergent $\varepsilon^{-1}$ terms remain the same

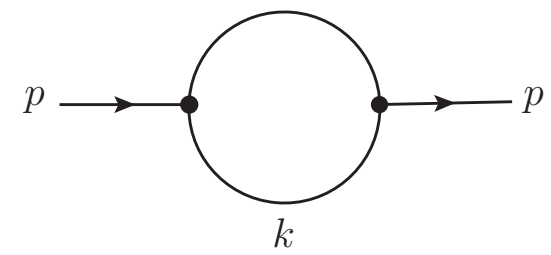

FIG. 1. The self-energy diagram for the scalar field in the $\phi^{3}$ theory in the one-loop approximation.

regardless of a value of $R_{c}$, in full accordance with the results of [34]. It is to be expected, since the compactification is an infrared process which can not change the UV properties of the theory.

\section{COMPACTIFICATION ON ORBIFOLD $S^{2} / Z_{2}$}

In Sec. III the manifold $M_{4} \otimes T^{2} / Z_{2}$ was studied. In this section we examine the case when the six-dimensional scalar field $\phi$ is defined on a manifold $M_{4} \otimes S^{2} / Z_{2}$, with a radius of two-dimensional sphere $S^{2}$ to be $R_{c}$. It is appropriate to introduce spherical coordinates $\theta, \phi$, and use the following expansion:

$$
\phi(x, \theta, \phi)=\frac{1}{R_{c}} \sum_{l=0}^{\infty} \sum_{m=-l}^{l} Y_{l}^{m}(\theta, \phi) \phi_{l m}(x),
$$

where $Y_{l}^{m}(\theta, \phi)(m=-l,-l+1, \ldots, l-1, l)$ are spherical harmonics [35]. They obey the orthogonality condition

$\int_{0}^{2 \pi} d \phi \int_{0}^{\pi} \sin \theta d \theta Y_{l}^{m}(\theta, \phi)\left[Y_{l^{\prime}}^{m^{\prime}}(\theta, \phi)\right]^{*}=\delta_{l l^{\prime}} \delta_{m m^{\prime}}$.

Using formula

$$
\int_{0}^{2 \pi} d \phi \int_{0}^{\pi} \sin \theta d \theta Y_{0}^{0}(\theta, \phi) Y_{l}^{m}(\theta, \phi) Y_{l}^{-m}(\theta, \phi)=\frac{(-1)^{m}}{\sqrt{4 \pi}}
$$

one can show that an effective four-dimensional coupling constant is

$$
\bar{g}_{4}=\frac{g}{\sqrt{4 \pi} R_{c}},
$$

for zero mode interaction. For interactions between zero mode and $\mathrm{KK}$ modes, a coupling constant is equal to $(-1)^{m} \bar{g}_{4}$. The masses of the KK excitations are known to be numerated by an integer $l=0,1,2, \ldots[17,18]$,

$$
m_{l}^{2}=m_{0}^{2}+\frac{l(l+1)}{R_{c}^{2}} .
$$

Let us consider zero-mode self-energy $\Sigma\left(p^{2}\right)$ in the oneloop approximation (Fig. 1). It is given by 


$$
\begin{aligned}
\Sigma\left(p^{2}\right)= & \frac{\alpha}{2} \Gamma(\varepsilon)(4 \pi)^{\varepsilon}\left(\bar{\mu} R_{c}\right)^{2 \epsilon} R_{c}^{-2} \\
& \times \sum_{l=0}^{\infty} \sum_{m=-l}^{l} \int_{0}^{1} d x[l(l+1)+c]^{-\varepsilon} \\
= & \frac{\alpha}{2} \Gamma(\varepsilon)(4 \pi)^{\varepsilon}\left(\bar{\mu} R_{c}\right)^{2 \epsilon} R_{c}^{-2} \int_{0}^{1} d x \sum_{l=0}^{\infty} \frac{2 l+1}{[l(l+1)+c]^{\varepsilon}},
\end{aligned}
$$

where $c$ is defined by Eq. (35). The series on the right-hand side can be represented as

$$
\begin{aligned}
\zeta_{t}(s ; c) & =\sum_{l=0}^{\infty} \frac{2 l+1}{[l(l+1)+c]^{s}} \\
& =\left.\frac{1}{1-s} \frac{d}{d \alpha} \sum_{l=0}^{\infty} \frac{1}{[l(l+1)+\alpha(2 l+1)+c]^{s-1}}\right|_{\alpha=0} .
\end{aligned}
$$

We have

$$
\begin{aligned}
\sum_{l=0}^{\infty} \frac{1}{[l(l+1)+\alpha(2 l+1)+c]^{s-1}} & =\sum_{l=0}^{\infty} \frac{1}{\left[(l+a)^{2}+q\right]^{s-1}} \\
& =\zeta_{t}(s ; a, q)
\end{aligned}
$$

where

$$
a=\frac{1}{2}+\alpha, \quad q=c-\frac{1}{4}-\alpha^{2},
$$

and an analytic expression for $\zeta_{t}(s ; a, q)$ is given by Eq. (A6). Note that $\left.[d q / d \alpha]\right|_{\alpha=0}=0$. For $c \gg 1$, we obtain form (70)-(72), and (A6) that

$$
\zeta_{t}(\varepsilon ; c)=-c^{1-\varepsilon}\left[1-\frac{1}{12 c}\right]+\mathrm{O}\left(c^{-2}\right)
$$

as $\varepsilon \rightarrow 0$. As a result, we come to expression (39) (up to unimportant finite constant).

The above consideration can be also applied to a calculation of the effective four-dimensional vertex for zero mode interaction in the one-loop approximation (Fig. 2). Taking into account that

$$
\zeta_{t}(1+\varepsilon ; c)=c^{-\varepsilon}\left[\frac{1}{\varepsilon}+\frac{1}{12 c}\right]+\mathrm{O}\left(c^{-2}\right)
$$

as $\varepsilon \rightarrow 0$, we reproduce formula (53) (up to a constant factor). All said above allows us to conclude that in the large $R_{c}$ region our main results do not depend on a topology of the two-dimensional compact manifold.

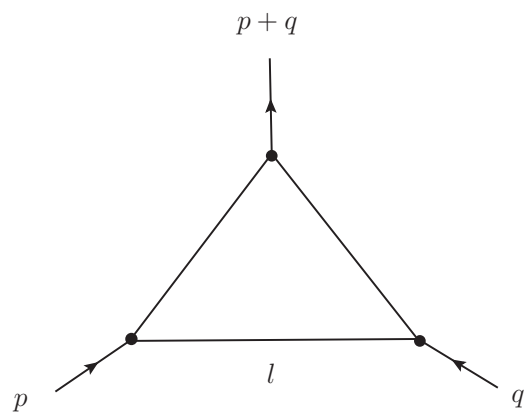

FIG. 2. Three-particle vertex in the scalar $\phi^{3}$ theory in the oneloop approximation.

\section{SCALE DEPENDENCE OF PHYSICAL OBSERVABLES}

As mentioned in Sec. III, a nontrivial dependence of physical quantities on the compactification radius appears when the physical scale $\left(\mu^{-1}\right.$, in our case) becomes much larger than $R_{c}$. In the opposite case, $\mu^{-1} \lesssim R_{c}$, when a physical process goes "inside a sphere of the radius $R_{c}$," such a dependence disappears.

Some other physical examples can be given which illustrate these statements. In [36] a generalization of the Froissart-Martin bound for scattering in $D$-dimensional spacetime with one compact dimension has been derived. The upper bound for the imaginary part of the hadronic scattering amplitude $T^{D}(s, t)$ was found to be

$$
\operatorname{Im} T_{D}(s, 0) \leq s R_{0}^{D-2}(s) \Phi\left(\frac{R_{0}}{R_{c}}, D\right) .
$$

In (75) the "transverse radius" is given by $R_{0}(s) \sim t_{0}^{-1 / 2} \ln s$, where $t_{0}$ denotes the nearest singularity in the $t$ channel. $R_{c}$ is the compactification radius of the $\mathrm{ED}$, and $\Phi\left(R_{0} / R_{c}, D\right)$ is a known function. At $R_{c} \gg R_{0}(s)$, the inequality (75) reproduces the Froissart-Martin bound in a flat spacetime with arbitrary $D$ dimensions [37]

$$
\sigma_{\text {tot }}^{D}(s) \leq \operatorname{const}(D) R_{0}^{D-2}(s)
$$

while in the opposite limit $R_{c} \ll R_{0}(s)$ it results in the inequality [36]

$$
\operatorname{Im} T^{D}(s, 0) \leq \operatorname{const}(D) s R_{0}^{D-3}(s) R_{c} .
$$

In [38] an analogous result has been obtained for the scattering of two SM particles on a 3D brane embedded into a flat spacetime with $n$ compact EDs $(D=4+n)$. The inelastic cross section $\sigma_{\text {in }}^{D}(s)$ was calculated in the transPlanckian region $\sqrt{s} \gg M_{D},|t|$, where $t$ is a momentum transfer squared, and $M_{D}$ is a fundamental Planck scale in $D$ dimensions. The result of the calculations is the following: 


$$
\sigma_{\text {inel }}^{D}(s) \simeq \operatorname{const}(D) \times \begin{cases}R_{0}^{2+n}(s), & R_{c} \gg \bar{R}_{0}(s), \\ R_{0}^{2}(s) R_{c}^{n}, & R_{c} \ll \bar{R}_{0}(s),\end{cases}
$$

where $\quad \bar{R}_{0}(s)=2 R_{g}(s) \sqrt{\ln \left(s / M_{D}^{2}\right)}, \quad R_{g}(s)$ being the "Regge gravitational radius" (for more details, see [38]).

To summarize, we can say that the dependence of physical observables on the compactification radius of the $\mathrm{ED}(\mathrm{s})$ arises only when the physical scale $R_{\text {phys }}$ of the process becomes larger than (comparable with) $R_{c}$. On the contrary, if $R_{\text {phys }} \ll R_{c}$, this dependence disappears (a physical process occurs on distances $\sim R_{\text {phys }}$, and it does not "feel" the large scale $R_{c}$ at all).

\section{CONCLUSIONS}

We have considered compactification of the asymptotically free $\phi_{D=6}^{3}$ theory to manifolds $M_{4} \otimes T^{2} / Z_{2}$ and $M_{4} \otimes S^{2} / Z_{2}$. The asymptotically free behavior of the dimensionless triple coupling in $M_{6}$ is being inherited by dimensional triple couplings of the light modes in both cases of compactification, with details depending of the shape of compactification. We also have considered the physical implications for high-energy scattering which has the same energy dependence as in simple four-dimensional case but retaining the compactification radius as a parameter, when the interaction radius exceeds the compactification size, while the "memory" of the latter disappears at short-distance interactions which has now a different energy dependence.

\section{APPENDIX: EPSTEIN'S INHOMOGENEOUS FUNCTION}

We give some useful properties of the two-dimensional inhomogeneous Epstein zeta function $Z_{2}^{a}(s)$ (34), with $a>0$. In [39] the following explicit expression was derived

$$
Z_{2}^{a}(s)=-a^{-s}+\frac{\pi a^{1-s}}{s-1}+\frac{A(s ; a)}{\Gamma(s)}
$$

where

$$
\begin{aligned}
A(s ; a)= & 4\left[a^{1 / 4}\left(\frac{\pi}{\sqrt{a}}\right)^{s} \sum_{n=1}^{\infty} n^{s-1 / 2} K_{s-1 / 2}(2 \pi n \sqrt{a})\right. \\
& +a^{1 / 2}\left(\frac{\pi}{\sqrt{a}}\right)^{s} \sum_{n=1}^{\infty} n^{s-1} K_{s-1}(2 \pi n \sqrt{a}) \\
& +\sqrt{2}(2 \pi)^{s} \sum_{n=1}^{\infty} n^{s-1 / 2} \sum_{d \| n} d^{1-2 s}\left(2+\frac{4 a}{d^{2}}\right)^{1 / 4-s / 2} \\
& \left.\times K_{s-1 / 2}\left(\pi n \sqrt{2+\frac{4 a}{d^{2}}}\right)\right] .
\end{aligned}
$$

Here $K_{\nu}(z)$ is the modified Bessel function of the second kind, $d \| n$ is the division of $n$. As one can see from (A2),
$A(s ; a)$ decreases exponentially, as $a \rightarrow \infty$. In the limit $a \rightarrow 0$ the Chowla-Selberg formula [40] takes place

$$
\begin{aligned}
Z_{2}(s)= & 2 \zeta(2 s)+2 \sqrt{\pi} \frac{\Gamma(s-1 / 2)}{\Gamma(s)} \zeta(2 s-1) \\
& +\frac{8 \pi^{s}}{\Gamma(s)} \sum_{n=1}^{\infty} n^{s-1 / 2} \sum_{d \| n} d^{1-2 s} K_{s-1 / 2}(2 \pi n),
\end{aligned}
$$

where

$$
Z_{2}(s) \equiv Z_{2}^{0}(s)=\sum_{n_{1}, n_{2} \in Z^{2}} \frac{1}{\left(n_{1}^{2}+n_{2}^{2}\right)^{s}}
$$

is the two-dimensional Epstein zeta function [41], and $\zeta(s)$ is the Riemann zeta function [32]. Note that all (multi) series in (A2), (A3) are exponentially convergent.

The above formulas are valid over the whole complex plane. The inhomogeneous Epstein function (A1) exibits an infinite number of simple poles at $s=1,1 / 2,-1 / 2,-3 / 2, \ldots$, while the homogeneous Epstein function (A3) has only two simple poles at $s=1$ and $s=1 / 2$, with the residues $\pi$ and $-1 / 2$, respectively [28]. Note that both functions are regular at $s=0$. The formula

$$
\begin{aligned}
\left.Z_{2}^{a}(s)\right|_{s \rightarrow 0}= & -a^{-s}+\frac{\pi a^{1-s}}{s-1} \\
& +\frac{4}{\Gamma(s)} \sum_{n=1}^{\infty} \frac{1}{n}\left[\frac{1}{2} e^{-2 \pi n \sqrt{a}}+\sqrt{a} K_{1}(2 \pi n \sqrt{a})\right. \\
& \left.+\sum_{d \| n} d e^{-\pi n \sqrt{2+4 a / d^{2}}}\right]+\mathrm{O}\left(s^{2}\right)
\end{aligned}
$$

gives an expansion of two-dimensional inhomogeneous Epstein function around the point $s=0$.

The truncated Epstein-like zeta function is given by the expression [39]

$$
\begin{aligned}
\zeta_{t}(s ; a, q)= & \sum_{n=0}^{\infty} \frac{1}{\left[(n+a)^{2}+q\right]^{s}} \\
= & \left(\frac{1}{2}-a\right) q^{-s}+\frac{q^{-s}}{\Gamma(s)} \sum_{m=1}^{\infty} \frac{(-1)^{m} \Gamma(m+s)}{m !} \\
& \times \zeta_{H}(-2 m ; a) q^{-m}+\frac{\sqrt{\pi} \Gamma(s-1 / 2)}{2 \Gamma(s)} q^{1 / 2-s} \\
& +\frac{2 \pi^{s}}{\Gamma(s)} q^{1 / 4-s / 2} \sum_{n=1}^{\infty} n^{s-1 / 2} \cos (2 \pi n a) \\
& \times K_{s-1 / 2}(2 \pi n \sqrt{q}),
\end{aligned}
$$

with $q>0$. The first series on the right-hand side is asymptotic. The last series decreases exponentially in parameter $q$. The quantity 


$$
\zeta_{H}(s ; a)=\sum_{n=0}^{\infty} \frac{1}{(n+a)^{s}}
$$

is a Hurwitz zeta function [32]. It is an analytic function over the entire complex $s$ plane except the point $s=1$, at which it has a simple pole. For $k=0,1,2, \ldots$, we have

$$
\zeta_{H}(-k ; a)=-\frac{B_{k+1}(a)}{k+1}
$$

where $B_{r}(a)$ is a Bernoulli polynomial [32]. In particular, $\zeta_{H}(0 ; a)=1 / 2-a$. In (A6), apart form the pole at $s=1 / 2$, there is a whole sequence of poles for $s=-1 / 2,-3 / 2, \ldots$.
[1] D. J. Gross and F. Wilzek, Ultraviolet Behavior of NonAbelian Gauge Theories, Phys. Rev. Lett. 30, 1343 (1973); Asymptotically free gauge theories. I, Phys. Rev. D 8, 3633 (1973).

[2] H. D. Politzer, Reliable Perturbative Results for Strong Interactions? Phys. Rev. Lett. 30, 1346 (1973).

[3] S. Coleman and D. J. Gross, Price of Asymptotic Freedom, Phys. Rev. Lett. 31, 851 (1973).

[4] A. D. Bond and D.F. Litim, Theorems for asymptotic safety of gauge theories, Eur. Phys. J. C 77, 429 (2017); Price of Asymptotic Safety, Phys. Rev. Lett. 122, 211601 (2019).

[5] D. J. Gross and A. Neveu, Dynamical symmetry breaking in asymptotically free field theories, Phys. Rev. D 10, 3235 (1974).

[6] E. Brezin and J. Zinn-Justin, Renormalization of the Nonlinear $\sigma$ Model in $2+\varepsilon$ Dimensions-Application to the Heisenberg Ferromagnets, Phys. Rev. Lett. 36, 691 (1976); E. Brezin, J. Zinn-Justin, and L. Le Guilou, Renormalization of the nonlinear $\sigma$ model in $2+\varepsilon$ dimensions, Phys. Rev. D 14, 2615 (1976).

[7] K. Symanzik, A field theory with computable largemomenta behaviour, Lett. Nuovo Cimento 6, 77 (1973).

[8] R. A. Brandt, Asymptotically free $\phi^{4}$ theory, Phys. Rev. D 14, 3381 (1976).

[9] A. J. Macfarlane and G. Woo, $\phi^{3}$ theory in six dimensions and the renormalization group, Nucl. Phys. B77, 91 (1974); Erratum, Nucl. Phys. B86, 548 (1975).

[10] E. Elizade and Yu. Kubyshin, Possible evidence of KaluzaKlein particles in a scalar model with spherical compactification, J. Phys. A 27, 7533 (1994).

[11] M. A. López-Osorio, E. Martínez-Pascual, G. I. NápolesCañedo, and J. J. Toscano, One-loop order effects from one universal extra dimension on $\lambda \phi^{4}$ theory, J. Phys. Commun. 4, 115004 (2020).

[12] R. Akhoury and C.S. Gauthier, Decoupling of heavy Kaluza-Klein modes in models with five-dimensional scalar fields, J. Phys. G 36, 015005 (2009).

[13] R. Akhoury and C. S. Gauthier, Kaluza-Klein model with spontaneous symmetry breaking: Light-particle effective action and its compactification scale dependence, Phys. Rev. D 78, 105002 (2008).

[14] E. Matrinez-Pascual, G. Nápoles-Cañedo, H. NovalesSánchez, A. Sierra-Martínez, and J. J. Toscano, Implications of extra dimensions in the effective charge and the beta function in quantum electrodynamics, Phys. Rev. D 101, 035034 (2020).
[15] C. Sochichiu, Quantum Kaluza-Klein compactification, Phys. Lett. B 463, 27 (1999).

[16] C. Sochichiu, Compactified quantum fields. Is there life beyond the cut-off scale? Phys. Lett. B 477, 253 (2000).

[17] H. Dohi and K. Oda, Universal extra dimensions on real projective plane, Phys. Lett. B 692, 114 (2010).

[18] N. Maru, T. Nomura, J. Sato, and M. Yamanaka, The universal extra dimensional model with $S^{2} / Z_{2}$ extra-space, Nucl. Phys. B830, 414 (2010).

[19] N. Maru, T. Nomura, and J. Sato, One-loop radiative correction to Kaluza-Klein mases in $S^{2} / Z_{2}$ universal extra dimensional model, Prog. Theor. Exp. Phys. 2014, 083 B04 (2014).

[20] H. Dohi, T. Kakuda, K. Nishiwaki, K.-y. Oda, and N. Okuda, Notes on sphere-based universal extra dimensions, Afr. Rev. Phys. 9, 0069 (2014), arXiv:1406.1954.

[21] T. Kakuda, K. Nishiwaki, and R. Watanabe, Universal extra dimensions after Higgs discovery, Phys. Rev. D 88, 035007 (2013).

[22] J. C. Collins, Renormalization (Cambridge University Press, Cambridge, England, 1984).

[23] B. P. Kosyakov, Physical sense of renormalizability, Phys. Part. Nucl. 32, 488 (2001), arXiv:hep-th/0011235.

[24] G. t'Hooft, Dimensional regularization and the renormalization group, Nucl. Phys. B61, 455 (1973).

[25] F. J. Yndurain, Quantum Chromodynamics. An Introduction to the Theory of Quarks and Gluons (Springer-Verlag, Berlin, 1983).

[26] A. N. Vasiliev, The Field Theoretic Renormalization Group in Critical Behavior Theory and Stochastic Dynamics (CRC Press Company, NY, 2020).

[27] M. Kompaniets and A. Pikelner, Critical exponents from five-loop scalar theory renormalization near six-dimensions, arXiv:2101.10018.

[28] K. Kirsten, Inhomogeneous multidimensional Epstein zeta function, J. Math. Phys. (N.Y.) 32, 3008 (1991); Generalized multidimensional Epstein zeta function, J. Math. Phys. (N.Y.) 35, 459 (1994).

[29] J. S. Dowker and R. Critchley, Effective Lagrangian and energy-momentum tensor in de Sitter space, Phys. Rev. D 13, 3224 (1976).

[30] S. W. Hawking, Zeta-function regularization of path integrals in curved space-time, Commun. Math. Phys. 55, 133 (1977).

[31] B. Zwiebach, A First Course in String Theory, 2nd ed. (Cambridge University Press, Cambridge, England, 2009). 
[32] Higher Transcendental Functions. Vol. 1. By the Staff of the Bateman Manuscript Project, edited by A. Erdélyi, W. Magnus, F. Oberhettinger, and F. G. Tricomi (McGrawHill Book Company, New York, 1953).

[33] D. V. Shirkov and I. L. Solovtsov, Analytic Model for the QCD Running Coupling with Universal $\bar{\alpha}_{s}(0)$ Value, Phys. Rev. Lett. 79, 1209 (1997); Ten years of the analytic perturbation theory in QCD, Theor. Math. Phys. 150, 132 (2007).

[34] M. J. Duff and D. J. Toms, Divergences and anomalies in Kaluza-Klein theories, CERN-3248, in Proceedings of the 2nd Seminar on Quantum Gravity, Moscow, USSR, edited by M. A. Markov and P. C. West (Plenum Press, New York, 1981), pp. 431-461.

[35] T. M. MacRobert, Spherical Harmonics: An Elementary Treatise on Harmonic Functions with Applications, 3rd ed. (Pergamon Press, New York, 1967).

[36] V. A. Petrov, Froissart-Martin bound in spaces with compact extra dimensions, Mod. Phys. Lett. A 16, 151 (2001); Froissart-Martin bound in spaces of compact dimensions, Phys. At. Nucl. 65, 877 (2002); High-energy behaviour in four and more dimensions, in Proceedings of the International Conference on Theoretical Physics (TH 2002), Paris, France (Birkhäuser Verlag, Basel, 2002), pp. 253-255.

[37] M. Chaichian and J. Fisher, Unitarity bounds for highenergy scattering in many dimensions, Phys. Appl. 14, 334 (1988); Higher-dimensional space-time and unitary bound on the scattering amplitude, Nucl. Phys. B303, 557 (1988).

[38] A. V. Kisselev and V. A. Petrov, Gravireggeons in extra dimensions and interaction of ultra-high energy cosmic neutrinos with nucleons, Eur. Phys. J. C. 36, 103 (2004).

[39] E. Elizade, Multidimensional extension of the generalized Chowla-Selberg formula, Commun. Math. Phys. 198, 83 (1998); Zeta functions: Formulas and applications, Comput. Appl. Math. 118, 125 (2000).

[40] S. Chowla and A. Selbergs, On Epstein's zeta function, Proc. Natl. Acad. Sci. U.S.A. 35, 317 (1949).

[41] P. Epstein, Zur Theorie allgemeiner Zetafunctionen, Math. Ann. 56, 615 (1903); Zur Theorie allgemeiner Zetafunctionen. II, Math. Ann. 63, 205 (1906). 